\title{
Electrodepósitos de Poli-3,4-etilendioxitiofeno sobre electrodos transparentes de Oxido de Indio y Estaño. Control del Espesor y Morfología.
}

ALEX PALMA-CANDO', MARGARITA RIVERA-HERNÁNDEZ², BERNARDO FRONTANA-URIBEI *

Centro Conjunto de Investigación en Química Sustentable UAEMex-UNAM Carretera Toluca-Atlacomulco km

14.5, Campus "San Cayetano", San Cayetano-Toluca, 50200, Estado de México, México.

2 Instituto de Física UNAM, México DF.

*Correspondencia: bafrontu@unam.mx

Recibido: 25 de abril de 2012 / Aceptado: 05 de octubre de 2013

\section{Resumen}

El presente estudio fue llevado a cabo con el motivo de obtener una curva que relacione la absorbancia (A) (a $\lambda$ $=700 \mathrm{~nm}$ ) con el espesor (L), obtenido directamente con la técnica de microscopía de fuerza atómica (AFM) en modo oscilante, de películas de poli-3,4-etilendioxitiofeno (PEDOT) depositado sobre electrodos transparentes de óxido de indio y estaño (ITO) mediante la técnica electroquímica de cronoamperometría (CA) a partir de una solución de EDOT $5 \mathrm{mM}$ / Perclorato de tetrabutilamonio (TBAP) 0.1M en acetonitrilo anhidro (AN). Se determinó el intervalo de absorbancia donde se tienen espesores de PEDOT sobre ITO, requeridos para utilizarse como capa extractora de huecos en celdas orgánicas solares (OPVd) entre 30 y $60 \mathrm{~nm}$. Se encontró que valores de A entre 0.1 a 0.2 dan como resultado películas de PEDOT del espesor requerido. La morfología de los distintos depósitos de PEDOT también fue analizada.

Palabras clave: PEDOT, ITO, espectrofotometría UV-vis, espesor, morfología, AFM.

\section{Electrodeposits of poly-3,4-ethylenedioxythiophene on indium tin oxide transparent electrodes. Thickness and morphology control}

\section{Abstract}

The current study was carried out in order to find a relation between absorbance $(A)(\lambda=700 \mathrm{~nm})$ and thickness (L) for deposits of poly-3,4-ethylenedioxithiophene (PEDOT) on transparent electrodes of indium tin oxide (ITO). The thickness was directly measured by atomic force microscopy (AFM) in tapping mode. The films were electrodeposited by chronoamperometry (CA) from $5 \mathrm{mM}$ EDOT solution in acetonitrile anhydrous (AN). $0.1 \mathrm{M}$ Tetrabutylammonium perchlorate (TBAP) was used as a supporting electrolyte. The absorbance range with a thickness between 30 and $60 \mathrm{~nm}$ was determined for PEDOT films. These types of films are suitable as hole extraction layers in organic photovoltaic devices (OPVd). It was found for absorbance values from 0.1 to 0.2 , it has PEDOT films of required thickness. Also, the morphology of the electropolymers was determined by tapping AFM.

Key words: PEDOT, ITO, UV-vis spectroscopy, thickness, morphology, AFM.

\section{Introducción}

El poli-3,4-etilendioxitiofeno (PEDOT) durante las pasadas dos décadas ha sido intensamente estudiado debido a su alta conductividad y excelente estabilidad [1], además, en su estado dopado tiene muy buena transparencia en la región UV-vis, por lo que se lo ha adoptado como el material extractor de huecos en celdas fotovoltaicas orgánicas (OPVd) en forma de PEDOT:PSS. La electropolimerización en solventes orgánicos elimina el problema de introducir material aislante (PSS) en la película conductora, resultando en el 
aumento de la eficiencia de la celda [2]. Otra ventaja de la electropolimerización, es el fácil control sobre el espesor (que deberá estar dentro de un intervalo de 30-60 nm para su uso en OPVd) [3], la morfología (que tiene un gran impacto en el área de contacto con la capa activa de OPVd) y la conductividad (manipulando el estado de dopaje) del depósito al controlar las distintas variables electroquímicas.

Una descripción detallada de la síntesis para el ácido 3,4-etilendioxitiofeno-2,5-dicarboxílico fue hecha por Gogte et al. en 1967. La descarboxilación de este intermediario llevó al EDOT y desde su introducción dentro de la química de los polímeros intrínsecamente conductores (ICPs) su producción industrial ha sido basada en la ruta de Gogte con cambios menores utilizando descarboxilación con catalizadores de cobre en el último paso [4].

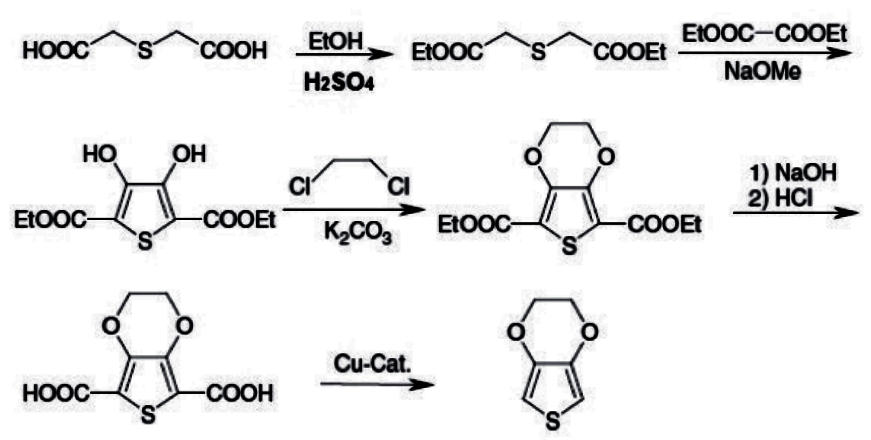

Figura 1. Síntesis de EDOT partiendo del oxalato de dietilo y del tiodigliocolato de dietilo

Para crear películas de PEDOT de interés para dispositivos prácticos, al menos cuatro diferentes métodos han sido empleados. Uno de ellos es la polimerización por vía electroquímica, donde el PEDOT puede ser fácilmente depositado sobre superficies conductoras (e.g. Pt, carbón vítreo, ITO, etc.) usando distintas técnicas electroquímicas. Tanto el agua como el acetonitrilo e inclusive ciertos líquidos iónicos orgánicos han demostrado ser útiles como solventes para la deposición electroquímica de PEDOT.

La deposición electroquímica puede ser hecha bajo corriente constante (método cronopotenciométrico), bajo potencial constante (método cronoamperométrico) o también mediante barridos cíclicos de potencial (método ciclo votamperométrico) al alcanzar un valor de potencial de oxidación donde el monómero genera radicales catión que a su vez polimerizan [5].

Las reacciones de electropolimerización usualmente se realizan en una celda de tres electrodos conectado a un potenciostato/galvanostato. Este arreglo consiste en un recipiente que permite la salida de gases del sistema, el ingreso de los reactantes, pero además, suficiente espacio para un electrodo de trabajo (WE) donde ocurre la reacción de interés, un contraelectrodo (CE) a través del cual circula la corriente y un electrodo de referencia (RE) respecto al cual se mide el potencial aplicado al electrodo de trabajo. La solución introducida en la celda de tres electrodos incluye al menos un disolvente apropiado para el electrolito soporte, que garantiza la conductividad del medio, como también de un monómero adecuado.

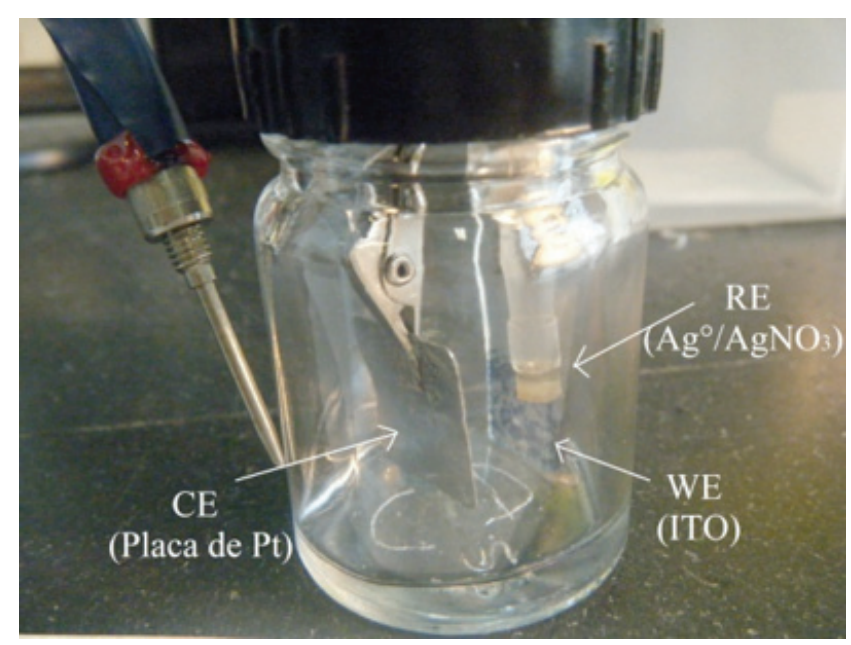

Figura 2. Celda de tres electrodos utilizada para la deposición de PEDOT sobre ITO

Los requerimientos experimentales no son extremos, se puede trabajar a temperaturas y presiones ambientales. La condición experimental más restrictiva es el requerimiento de una atmósfera inerte, en estos casos se logra con burbujeo directo de nitrógeno o argón puros (previamente humedecidos con el solvente utilizado para evitar cambios en la concentración) a la solución, por varios minutos, para desplazar al oxígeno disuelto.

La electropolimerización de PEDOT fue llevada a cabo en primer lugar por Dietrich en 1994 [6], y en los últimos años ha surgido el interés como un método alternativo a la deposición de películas por recubrimiento (e.g., inmersión, centrifugado, etc.). 
Además se ha mostrado que las características electroquímicas de películas de PEDOT electrodepositado son mucho mejores que las de PEDOT:PSS usado comúnmente en las OPVd [7], ya que este último incorpora material aislante PSS a la película conductora.

La necesidad de desarrollar fuentes de energía renovables a bajo costo ha introducido el potencial de obtener métodos baratos y sencillos de producir energía a partir de la luz solar con la ayuda de las OPVd. Un tipo de OPVd es el llamado dispositivo de heterounión en masa (BHJ-OPVd), en el que se utiliza un sustrato conductor transparente, ITO generalmente, como ánodo y sobre este una capa de PEDOT, obtenido vía química o electroquímicamente. Esta capa de PEDOT:PSS (clásicamente utilizada) mejora la calidad de la superficie del electrodo de ITO (e.g. aumentando el tiempo de vida del dispositivo, reduciendo la probabilidad de cortos) así como facilita la extracción de huecos (Fig. 3). Por otra parte, la función trabajo de este electrodo puede ser modificado por reacciones redox químicas - electroquímicas de la capa de PEDOT. Mezclar polímeros conjugados (e.g. P3HT, MDMO-PPV, etc.) con aceptores de electrones (e.g. fullerenos), es una muy eficiente vía para romper excitones fotoexcitados en portadores de carga libres (electrones y huecos). Por último un contacto metálico de aluminio evaporado, como cátodo, es colocado para cerrar el circuito [8].

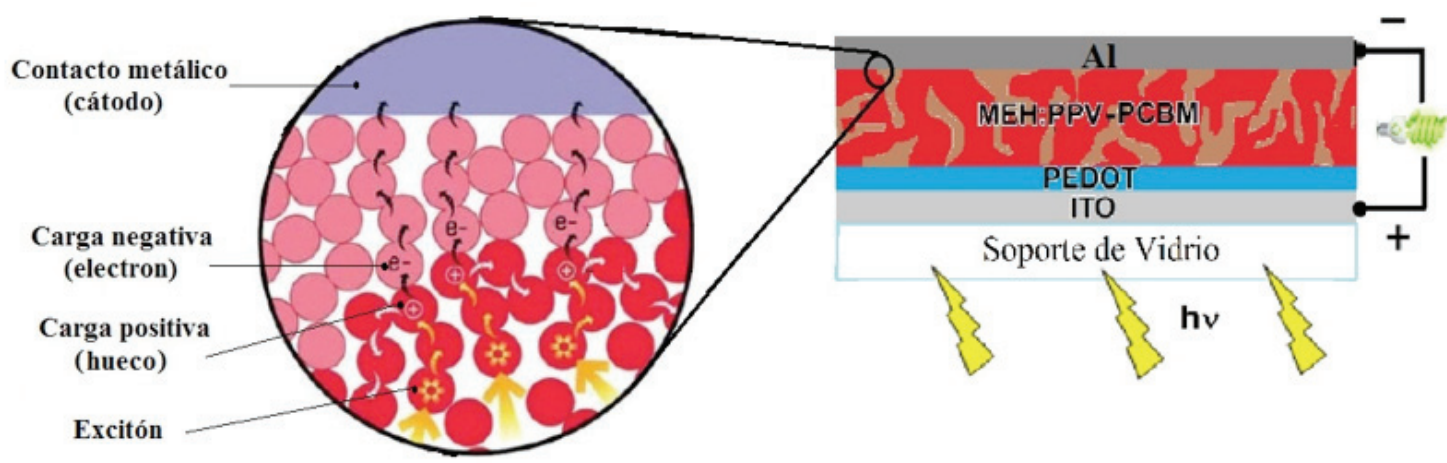

Figura 3. Esquema de una BHJ-OPVd y proceso de separación y extracción de electrones y agujeros.

Se ha mostrado que al controlar el espesor, se controla en parte la morfología de la película de polímero, misma que tiene gran impacto en la eficiencia de una OPVd [2], observándose que las mejores propiedades conductoras se tienen a espesores de 30 a $60 \mathrm{~nm}$ (en algunos casos hasta $100 \mathrm{~nm}$ ), para su uso en OPVd $[2,3,7,9,10]$. Con espesores de este orden se tiene una capa de PEDOT en contacto con el electrodo de ITO totalmente densa y una superficie rugosa, pero de gránulos pequeños $( \pm 10 \mathrm{~nm})$ previniendo así cortos circuitos en la OPVd [2]. Además, se ha observado que es posible relacionar el espesor de las películas de PEDOT (obtenidas generalmente por métodos como SEM o perfilométricos los mismos que nos dan una medida directa del grosor pero son algo complicadas sus mediciones y/o conllevan mucho tiempo en las lecturas) con la absorbancia UV-vis [2] a una cierta longitud de onda, siendo esta última una técnica muy fácil, rápida y económica.
El espesor (y a su vez la morfología) de una película se la puede medir directamente con la técnica de AFM, al hacer un escalón en la película (e.g., con la ayuda de una navaja), y hacer una medición en dicha zona [1 1]. Mientras que la curva de absorbancia se la puede obtener en un espectrofotómetro UV-vis, debido a la buena transparencia del PEDOT en su estado dopado.

\section{Parte experimental}

\subsection{Limpieza de los electrodos de ITO}

Todos los electrodos de ITO nuevos (KINTEC, $10 \mathrm{ohm/}$ $\mathrm{sq}$, Polished grade, $25 \times 10 \times 1.1 \mathrm{~mm}$ ) fueron inicialmente limpiados con un trapo suave empapado con solución acuosa de Tritón X-100 (1:100), y subsecuentemente limpieza ultrasónica en soluciones sucesivas de Tritón X-100, agua destilada, etanol y EDTA $1 \mathrm{mM}$ $(\mathrm{pH}=13)$ por 10 min cada una, enjuagando con abun- 
dante cantidad de agua destilada después de cada paso. En diversos ensayos se reutilizó los electrodos de ITO, previamente depositados con PEDOT, recuperándolos con la ayuda de un pedazo de algodón empapado con clorobenceno, se frotó firmemente al electrodo hasta remoción total del mismo, se enjuagó con acetona y se secó, para finalmente aplicar el procedimiento de limpieza previamente indicado. Tanto las propiedades ópticas como las electroquímicas fueron monitoreadas, observándose la no existencia de cambios sustanciales en estos electrodos recuperados al compararlos con los de un electrodo nuevo y limpio.

\subsection{Depósito de PEDOT/ $/ \mathrm{ClO}_{4}{ }^{-}$sobre ITO mediante CA}

Se preparó una solución de EDOT (ALDRICH, $\left.P M=142.15 \mathrm{~g} / \mathrm{mol}, \quad d=1.334 \mathrm{~g} / \mathrm{cm}^{3}\right) \quad 5 \mathrm{mM} / \quad$ TBAP (FLUKA, $\geq 99 \%$ pureza, $P M=341.92 \mathrm{~g} / \mathrm{mol}$ ) $0.1 \mathrm{M}$ en AN anhidro (SIGMA-ALDRICH, 99.8\% pureza) bajo atmosfera de $\mathrm{N}_{2}$ (INFRA Ultra alta pureza $99.999 \%$, burbujeo por $10 \mathrm{~min}$ ) a $25^{\circ} \mathrm{C}$ (con la ayuda de un baño térmico). La solución se prepara dentro de una celda de 3 electrodos (como la mostrada en la Fig. 2) en atmosfera de $\mathrm{N}_{2}$, teniendo como WE: ITO lárea de depósito $\approx 1.5 \times 1.0 \mathrm{~cm}$ ), CE: placa de Pt (área de $1.8 \times 1.0 \mathrm{~cm}$ ), paralelamente dispuestos a una distancia de $1.5 \mathrm{~cm}$, y RE: $\mathrm{Ag}^{\circ} / \mathrm{AgNO}_{3}\left(\mathrm{AgNO}_{3} 0.01 \mathrm{M} /\right.$ TBAP $0.1 \mathrm{M}$ en AN, $0.47 \mathrm{~V}$ vs. ENH). Con la ayuda de un potenciostato BAS100W se aplicó la técnica electroquímica $\mathrm{CA}$, teniendo como parámetros: Potencial inicial, Ei=-1400 mV (aplicado por 6s antes de la medición), potencial de oxidación, $\mathrm{E}_{\mathrm{Ox}_{\mathrm{x}}}=1311 \mathrm{mV}, \mathrm{y}$ tiempos de pulso de 1, 2, 2.5, 3.5, 4.5, 5, 10, 20 y $30 \mathrm{~s}$. Finalmente el electrodo depositado con polímero se enjuagó con $A N$, se secó con una corriente de $N_{2}$ y se lo guardó en viales herméticos con atmosfera inerte hasta posterior medición del espectro UV-vis y espesor en AFM. Los experimentos se repitieron por triplicado de manera aleatoria en ensayos independientes.

\subsection{Medición del espectro UV-vis para películas de PEDOT $/ \mathrm{ClO}_{4}^{-}$sobre ITO}

Los electrodos de ITO:PEDOT/ $\mathrm{ClO}_{4}^{-}$en su estado oxidado a $1311 \mathrm{mV}$, se introdujeron en una celda adaptada para un espectrofotómetro UV-vis (THERMO SCIENTIFICGENESYS IOS UV-vis) y se corrió el espectro de 300 a $1100 \mathrm{~nm}$ para cada uno de los depósitos obtenidos a distintos tiempos de depósito, se registró el valor de absorbancia a longitud de onda de 700 nm para relacionarlo posteriormente con el espesor de las películas obtenidas por mediciones de AFM.

\subsection{Medición del espesor de las películas de PE- $\mathrm{DOT} / \mathrm{ClO}_{4}^{-}$sobre ITO mediante AFM en modo oscilante.}

Con la ayuda de un equipo de AFM (JEOL JSPM 4210 Scanning Probe Microscope) en modo oscilante con un escáner de $80 \times 80 \mu \mathrm{m}$ se realizaron las mediciones de los espesores de los depósitos de PE$\mathrm{DOT} / \mathrm{ClO}_{4}^{-}$sobre ITO. Para tal efecto, se realizó un corte transversal en los electrodos con la ayuda de una navaja de acero inoxidable e inmediatamente se llevó al equipo de AFM con el propósito de medir la altura del escalón formado. Se empezó observando el depósito con un campo de $50 \mu^{2}{ }^{2}$ hasta encontrar el escalón, luego de esto se realizó una ampliación a $20 \mu^{2}$ a la zona de interés. Las imágenes se procesaron para obtener varios perfiles de la película y con esto alturas del depósito estudiado (utilizadas en el análisis estadístico) y la imagen 3D de la morfología del electropolímero.

\section{Resultados y discusión}

El primer estudio que se debe realizar para cualquier electropolimerización consiste en encontrar tanto la ventana de electroactividad del medio (VE) como la ventana de trabajo de electropolimerización (VT). Para lo primero, se utiliza la celda de tres electrodos y una solución libre de monómero, se realizan CV empezando con un intervalo de potencial pequeño (e.g., de $-500 \mathrm{mV}$ hasta $500 \mathrm{mV}$ ) y se va incrementando el potencial hacia cada lado con el fin de encontrar las barreras de reducción y oxidación de nuestro medio de trabajo. Luego de esto se añade el monómero con el cual se piensa trabajar (EDOT $5 \mathrm{mM}$ ) y según el intervalo de potencial hallado previamente se corre sendos CV con la finalidad de observar el comportamiento de nuestro monómero dentro de estos límites y de esta manera poder establecer los potenciales con los cuales podemos trabajar.

La siguiente figura muestra tanto la VE como la VT para el sistema utilizado en nuestro estudio: 


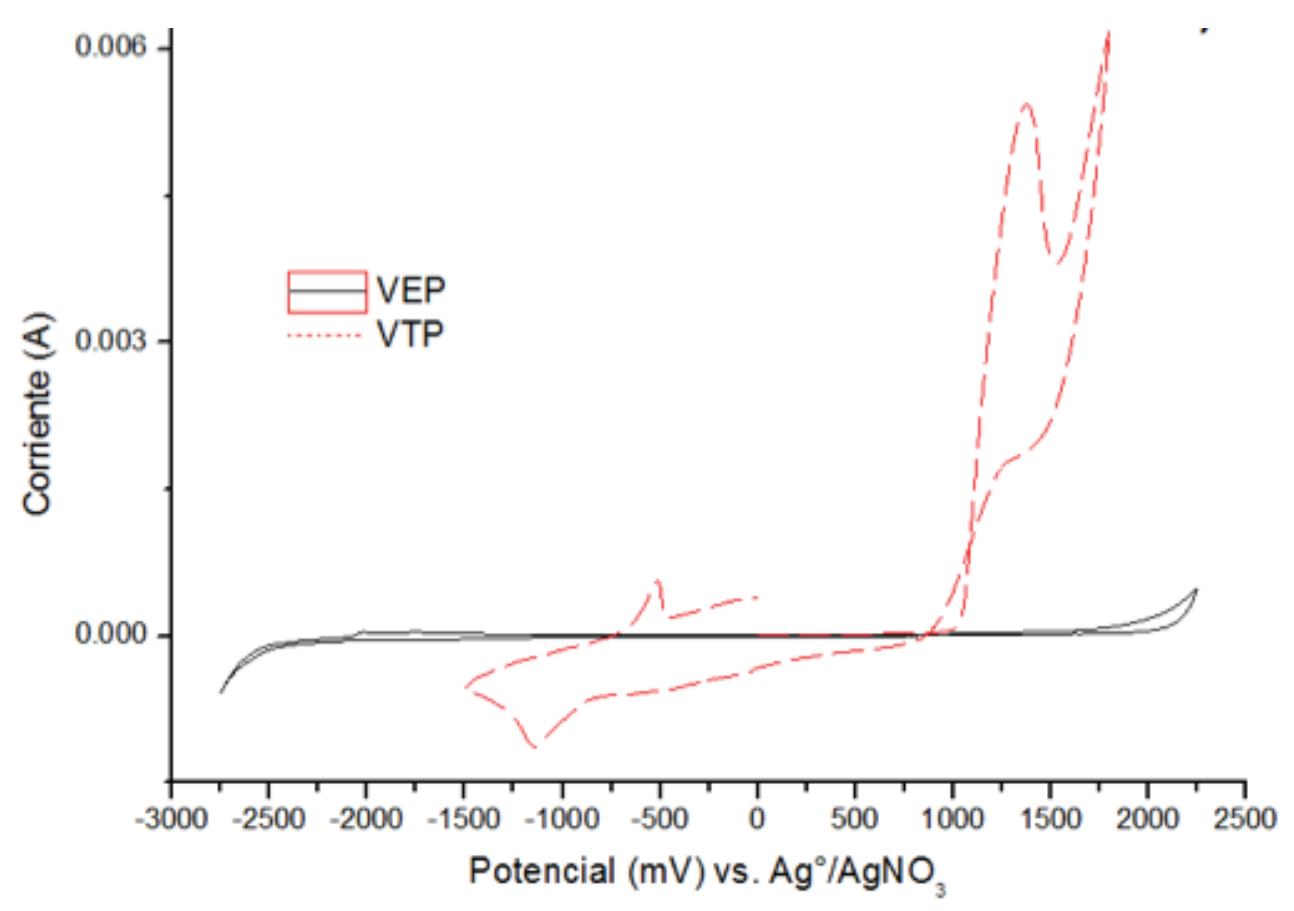

Figura 4. VE para sol. TBAP $0.1 \mathrm{M}$ en $A N$ y VT para sol.

EDOT $5 \mathrm{mM} /$ TBAP $0.1 \mathrm{M}$ en AN.

La VE es de más o menos $4.5 \mathrm{~V}$. La forma del primer $\mathrm{CV}$ muestra el típico comportamiento con un muy rápido incremento de la corriente anódica en el pico de oxidación del monómero [12] y observándose claramente el cruce de la curva en el barrido inverso debido al llamado "nucleation loop" que se origina por la nucleación del monómero sobre el electrodo desnudo [13]. Además, se tiene un potencial de pico de alrededor $1.375 \mathrm{~V}$ vs. $\mathrm{Ag}^{\circ} / \mathrm{AgNO}_{3}\left(\mathrm{AgNO}_{3} 0.01 \mathrm{M} / \mathrm{TBAP}\right.$ $0.1 \mathrm{M}$ en $\mathrm{AN}, 0.47 \mathrm{~V}$ vs ENH).
Una vez que se conoció el comportamiento electroquímico de nuestro sistema se procedió a realizar los depósitos de PEDOT/ $/ \mathrm{ClO}_{4}{ }^{-}$sobre ITO mediante $\mathrm{CA}$, con el fin de obtener la relación entre absorbancia a $\lambda=$ $700 \mathrm{~nm}$ y espesor (se tomó el valor de absorbancia a esta longitud de onda, debido a que se presenta un hombro en el espectro UV-vis del depósito en esta región, teniendo una buena correlación entre el espesor y la absorbancia a dicha longitud de onda). El espectro UV-vis de depósitos de PEDOT/ClO ${ }_{4}^{-}$tiene la siguiente forma, idéntica a las reportadas en la literatura [2].

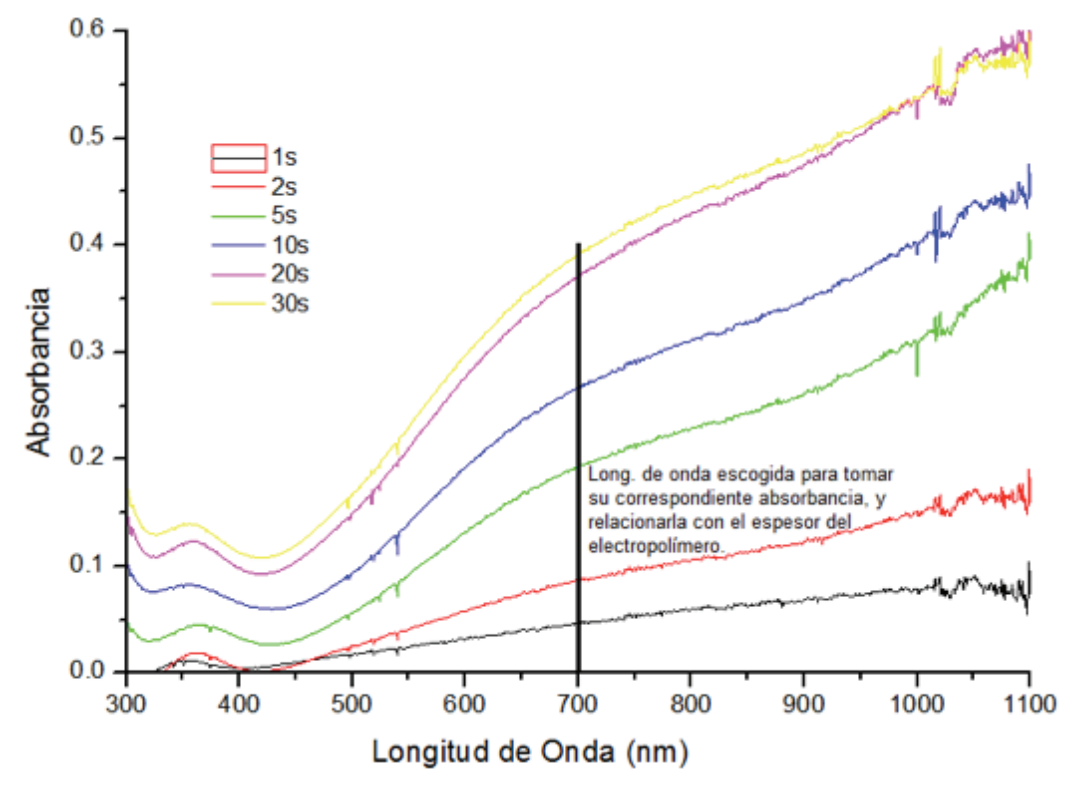

Figura 5. Espectros UV-vis de depósitos de PEDOT/ $\mathrm{ClO}_{4}^{-}$por CA a distintos tiempos de pulso. 
Con la técnica de AFM en modo oscilante se halló el espesor de cada depósito y se observó la morfología de los depósitos de PEDOT/ $/ \mathrm{ClO}_{4}{ }^{-}$sobre ITO a los distintos tiempos de pulso. Como se puede observar en la Fig. 6, la topografía para todos los depósitos muestra forma de gránulos típico de los depósitos de PEDOT teniendo como solvente AN [3,7]. Se sabe que para tener la mayor eficiencia de conversión energética, el depósito de capa extractora de huecos debe ser densa (sobre el ITO) pero a su vez presentar la mayor área de superficie posible en contacto con la capa activa, por lo que la topografía que presentan estas películas de PEDOT contribuye al buen contacto de las capas. Un problema se presenta cuando el tamaño de los cúmulos es demasiado grande (> $100 \mathrm{~nm}$ ), ya que se puede presentar cortos en la celda, disminuyendo así la PCE. Se puede establecer que a tiempos altos de depósito, $30 \mathrm{~s}$ se tiene formación de cúmulos anchos y demasiado altos ( $\pm 200 \mathrm{~nm}$ ) que podrían ocasionar cortos en la OPVd. A tiempos cortos de depósito, $1 \mathrm{~s}$ se puede observar un depósito poco denso y poco homogéneo, que de igual manera no presentaría un buen desempeño. A tiempos intermedios, $5 \mathrm{~s}$ se puede observar una capa densa de PEDOT pero a su vez una superficie muy rugosa la misma que tiene alturas < $100 \mathrm{~nm}$, presentando condiciones ideales para su uso como capa extractora de huecos en OPVd.

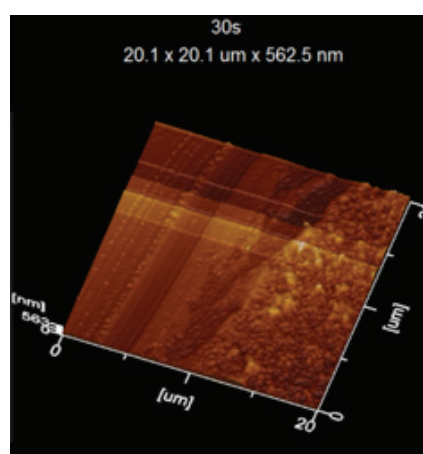

$10 \mathrm{~s}$

$21.7 \times 21.7 \mathrm{um} \times 682.0 \mathrm{~nm}$

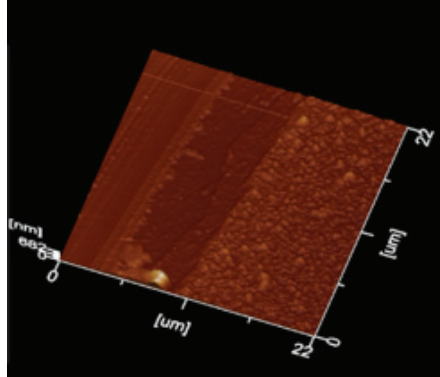

25

$21.3 \times 21.3 \mathrm{um} \times 276.5 \mathrm{~nm}$

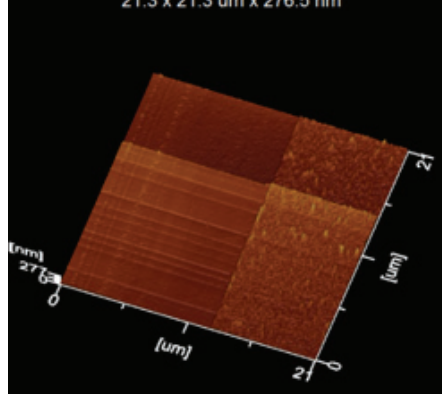

$20.0 \times 20.0 \mathrm{um} \times 851.1 \mathrm{~nm}$

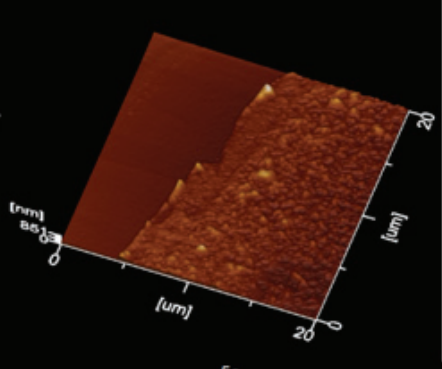

5 s
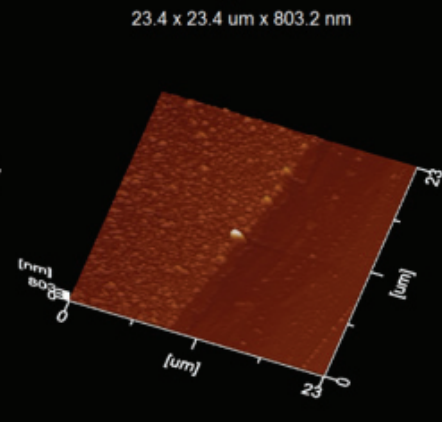

1 s

$13.0 \times 13.0 u m \times 665.9 \mathrm{~nm}$

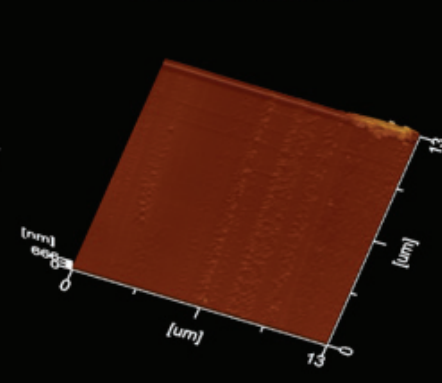

Figura 6. Imágenes 3D de AFM en modo oscilante de depósitos de PEDOT/CIOsobre ITO, obtenidos mediante CA utilizando diversos tiempos de pulso. 
A partir de estos resultados iniciales se pudo establecer que el espesor requerido para obtener depósitos de 30 a $60 \mathrm{~nm}$ se hallaba a tiempos de pulso por debajo de $5 \mathrm{~s}$ (espesor de $60.2 \pm 11.9 \mathrm{~nm}$ ), por lo que se realizó nuevos depósitos a tiempos menores, 2.5, 3.5 y $4.5 \mathrm{~s}$.
El análisis de altura de escalón y la morfología para los últimos depósitos se muestran en la Fig. 7. Los depósitos presentan una capa densa pero rugosa, con altura de cúmulos de $\pm 80 \mathrm{~nm}$, y el espesor de las películas dentro del intervalo requerido de 30 a $60 \mathrm{~nm}$.

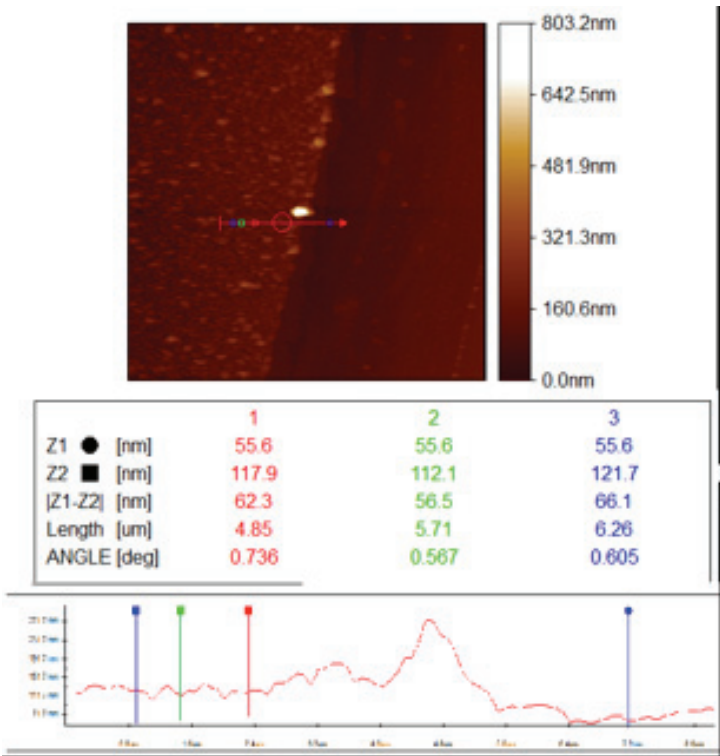

$3.5 \mathrm{~s}$

$21.5 \times 21.5 \mathrm{um} \times 557.4 \mathrm{~nm}$

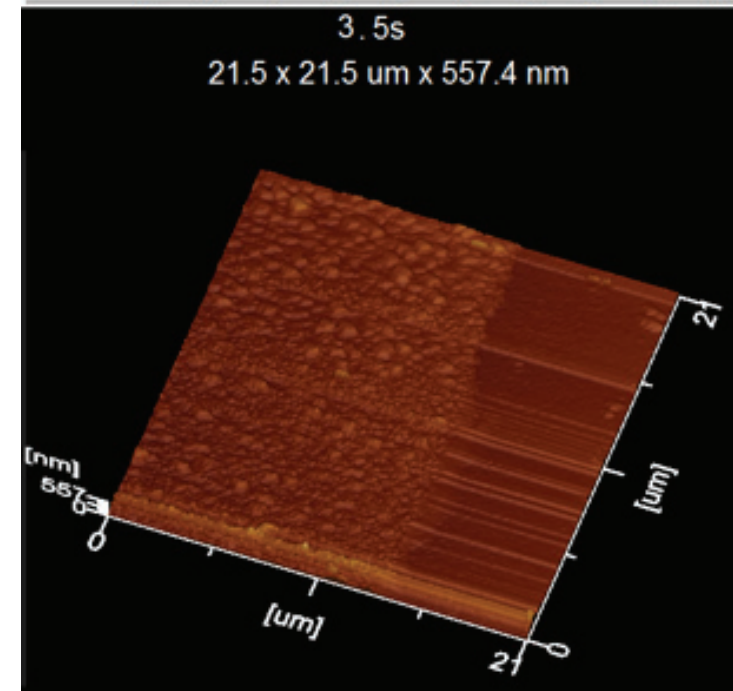

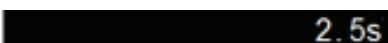

$21.3 \times 21.3 \mathrm{um} \times 274.6 \mathrm{~nm}$

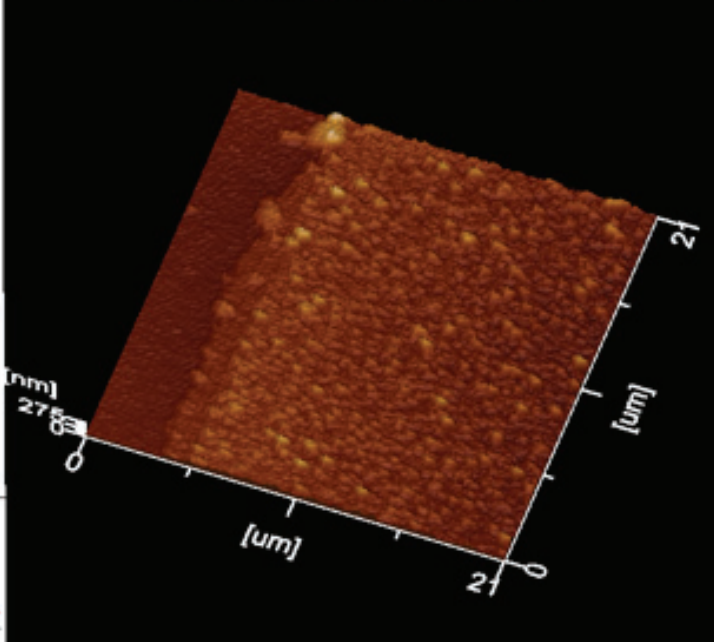

4_5sT2

$21.5 \times 21.5 \mathrm{um} \times 699.7 \mathrm{~nm}$

Figura 7. Imágenes de $A F M$ en modo oscilante para depósitos a tiempo intermedios, y análisis de escalón para un depósito PEDOT/ClO ${ }_{4}^{-}$de $5 \mathrm{~s}$.

Con el análisis estadístico de los resultados obtenidos de absorbancia mediante espectrofotometría UV-vis y de los espesores con la ayuda de mediciones de AFM en modo oscilante, se pudo obtener la siguiente relación válida para espesores menores a 100 nm, como se observa en la Fig. 8b: 


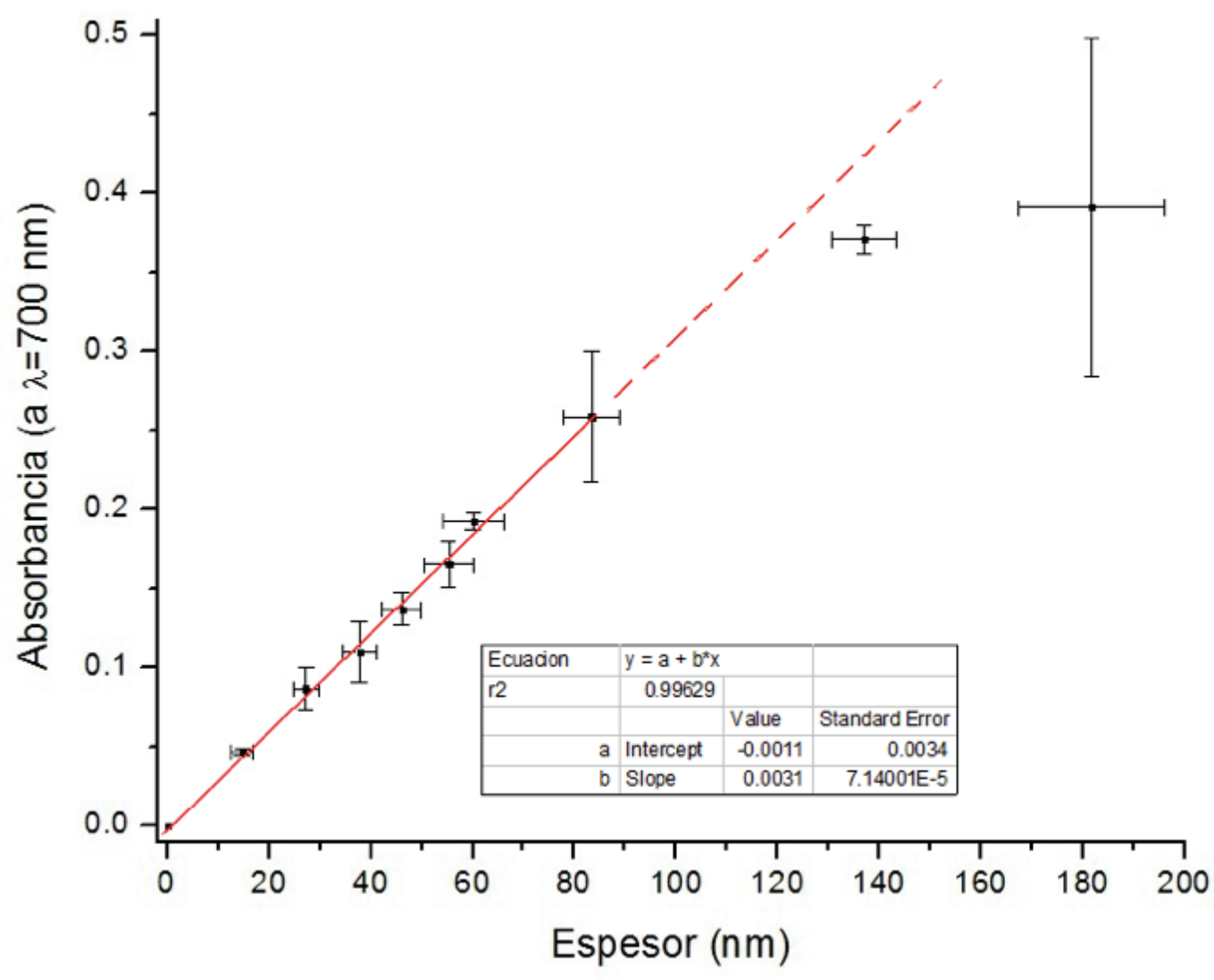

Figura 8. Absorbancia (a $\lambda=700 \mathrm{~nm}$ ) en función del espesor de películas de PEDOT/ $\mathrm{ClO}_{4}^{-}$sobre ITO depositadas mediante CA a tiempos de pulso de 1 a 30 s con $\alpha=0.05$ y regresión lineal para tiempos de pulso de 1 a $10 \mathrm{~s}$.

Se puede establecer para películas de PEDOT/ $\mathrm{ClO}_{4}$ sobre ITO depositadas por CA, a absorbancias de 0.1 a 0.2 se tiene espesores dentro del intervalo requerido de 30 a $60 \mathrm{~nm}$, útiles como capa extractora de huecos en OPVd. La mayor importancia de esta relación radica en la facilidad con que se puede establecer el espesor de una película de PEDOT/ClO ${ }_{4}^{-}$(dopada a $1311 \mathrm{mV}$ vs. $\mathrm{Ag}^{\circ} / \mathrm{AgNO}_{3}$ ) obtenida por $\mathrm{CA}$, teniendo únicamente la absorbancia de la misma a $\lambda=700 \mathrm{~nm}$, con buena exactitud, evitando de esta manera el uso de técnicas más complejas, a veces poco disponibles y demandantes de tiempo como son SEM o perfilométricas.

\section{Conclusiones}

En este trabajo se ha podido determinar una ecuación que relaciona la absorbancia de películas de PE-
DOT/ $\mathrm{ClO}_{4}{ }^{-}$sobre ITO, obtenidas mediante $\mathrm{CA}$, con el espesor de las mismas de grosores útiles para la elaboración de dispositivos fotovoltaicos. También se determinó que la morfología que presentan las películas con espesores dentro de 30 a 60 nm, sería la más adecuada para su aplicación en BHJ-OPVd ya que se tiene una capa densa con una superficie muy rugosa pero de tamaño de cúmulos $<100 \mathrm{~nm}$.

\section{Agradecimientos}

Al Ing. Mario Monroy por su colaboración en la obtención de las imágenes en el equipo de AFM del Instituto de Física de la UNAM. A. Palma-Cando quiere agradecer al Gobierno de México y la Secretaria de Relaciones Exteriores por el otorgamiento de la beca No. 7446. 


\section{Referencias}

[1] Groenendaal, L., Jonas, F., Freitag, D., Pielartzik, H., \& Reynolds, J. R. (2000). Poly(3,4-ethylenedioxythiophene) and Its Derivatives: Past, Present, and Future. Advanced Materials, 12(7), 481-494.

[2] Nasybulin, E., Wei, S., Cox, M., Kymissis, I., \& Levon, K. (2011). Morphological and Spectroscopic Studies of Electrochemically Deposited Poly(3,4-ethylenedioxythiophene) (PEDOT) Hole Extraction Layer for Organic Photovoltaic Device (OPVd) Fabrication. The Journal of Physical Chemistry C, 115(10), 4307-4314.

[3] Armstrong, N. R., Carter, C., Donley, C., Simmonds, A., Lee, P., Brumbach, M., . . Yoo, S. (2003). Interface modification of ITO thin films: organic photovoltaic cells. Thin Solid Films, 445(2), 342-352.

[4] Reuter, K., Kirchmeyer, S., \& Elschner, A. (2009). PEDOT - Properties and Technical Relevance. In I. F. Perepichka \& D. F. Perepichka (Eds.), Handbook of Thiophene-based Materials: Applications in Organic Electronics and Photonics: Wiley.

[5] Inzelt, G. (Ed.) (2008) Electrochemical Dictionary. Springer.

[6] Dietrich, M., Heinze, J., Heywang, G., \& Jonas, F. (1994). Electrochemical and spectroscopic characterization of polyalkylenedioxythiophenes. Journal of Electroanalytical Chemistry, 369(102), 87-92.

[7] Lee, H. J., Lee, J., \& Park, S.-M. (2010). Electrochemistry of Conductive Polymers. 45. Nanoscale Conductivity of PEDOT and PEDOT:PSS Composite Films Studied by Current-Sensing AFM. The Journal of Physical Chemistry B, 114(8), 2660-2666.

[8] Günes, S., Neugebauer, H., \& Sariciftci, N. S. (2007). Conjugated Polymer-Based Organic Solar Cells. Chemical Reviews, 107(4), 13241338.

[9] Rider, D. A., Harris, K. D., Wang, D., Bruce, J., Fleischauer, M. D., Tucker, R. T., . . Buriak, J. M. (2008). Thienylsilane-Modified Indium Tin Oxide as an Anodic Interface in Polymer/Fullerene Solar Cells. ACS Applied Materials \& Interfaces, 1(2), 279-288.

[10] Pringle, J. M., Armel, V., \& MacFarlane, D. R. (2010). Electrodeposited PEDOT-on-plastic cathodes for dye-sensitized solar cells. Chemical Communications, 46(29), 5367-5369.

[11] Wakizaka, D., Fushimi, T., Ohkita, H., \& Ito, S. (2004). Hole transport in conducting ultrathin films of PEDOT/PSS prepared by layer-bylayer deposition technique. Polymer, 45(25), 8561-8565.

[12] Vorotyntsev, M. A., Zinivyeva, V. A., \& Konev, D. V. (2010). Mechanisms of Electropolymerization and Redox Activity: Fundamental Aspects. In S. Cosnier \& A. Karyakin (Eds.), Electropolymerization: Concepts, Materials and Applications: WILEY-VCH.

[13] Heinze, J., Rasche, A., Pagels, M., \& Geschke, B. (2007). On the Origin of the So-Called Nucleation Loop during Electropolymerization of Conducting Polymers. The Journal of Physical Chemistry B, 111(5), 989-997. 